\title{
BMJ
}

\section{Effect of high flow oxygen on mortality in chronic obstructive pulmonary disease patients in prehospital setting: randomised controlled trial}

\author{
Michael A Austin, honorary associate, ${ }^{1}$ emergency medicine registrar, ${ }^{2}$ wilderness helicopter, intensive care \\ paramedic, ${ }^{3}$ Karen E Wills, biostatistician, ${ }^{1}$ Leigh Blizzard, senior biostatistician, ${ }^{1}$ Eugene $\mathrm{H}$ Walters, \\ professorial fellow, ${ }^{1}$ Richard Wood-Baker, honorary fellow, ${ }^{1}$ director ${ }^{2}$
}

Menzies Research Institute, University of Tasmania, Hobart, Tasmania, 7001 Australia

${ }^{2}$ Department of Respiratory Medicine, Royal Hobart Hospital, Hobart, Tasmania

${ }^{3}$ Tasmanian Ambulance Service, Hobart, Tasmania

Correspondence to: M A Austin maaustin@utas.edu.au

Cite this as: $B M J$ 2010;341:C5462 doi:10.1136/bmj.c5462

\section{ABSTRACT}

Objectives To compare standard high flow oxygen treatment with titrated oxygen treatment for patients with an acute exacerbation of chronic obstructive pulmonary disease in the prehospital setting.

Design Cluster randomised controlled parallel group trial. Setting Ambulance service in Hobart, Tasmania, Australia.

Participants 405 patients with a presumed acute exacerbation of chronic obstructive pulmonary disease who were treated by paramedics, transported, and admitted to the Royal Hobart Hospital during the trial period; 214 had a diagnosis of chronic obstructive pulmonary disease confirmed by lung function tests in the previous five years.

Interventions High flow oxygen treatment compared with titrated oxygen treatment in the prehospital (ambulance) paramedic) setting.

Main outcome measure Prehospital or in-hospital mortality.

Results In an intention to treat analysis, the risk of death was significantly lower in the titrated oxygen arm compared with the high flow oxygen arm for all patients (high flow oxygen $n=226$; titrated oxygen $n=179$ ) and for the subgroup of patients with confirmed chronic obstructive pulmonary disease (high flow $n=117$; titrated $\mathrm{n}=97$ ). Overall mortality was $9 \%$ (21 deaths) in the high flow oxygen arm compared with $4 \%$ (7 deaths) in the titrated oxygen arm; mortality in the subgroup with confirmed chronic obstructive pulmonary disease was $9 \%$ (11 deaths) in the high flow arm compared with $2 \%$ (2 deaths) in the titrated oxygen arm. Titrated oxygen treatment reduced mortality compared with high flow oxygen by $58 \%$ for all patients (relative risk $0.42,95 \%$ confidence interval 0.20 to $0.89 ; \mathrm{P}=0.02$ ) and by $78 \%$ for the patients with confirmed chronic obstructive pulmonary disease $(0.22,0.05$ to $0.91 ; \mathrm{P}=0.04)$. Patients with chronic obstructive pulmonary disease who received titrated oxygen according to the protocol were significantly less likely to have respiratory acidosis (mean difference in $\mathrm{pH} 0.12$ (SE 0.05); $\mathrm{P}=0.01 ; \mathrm{n}=28$ ) or hypercapnia (mean difference in arterial carbon dioxide pressure $-33.6(16.3) \mathrm{mm} \mathrm{Hg} ; \mathrm{P}=0.02 ; \mathrm{n}=29)$ than were patients who received high flow oxygen.

Conclusions Titrated oxygen treatment significantly reduced mortality, hypercapnia, and respiratory acidosis compared with high flow oxygen in acute exacerbations of chronic obstructive pulmonary disease. These results provide strong evidence to recommend the routine use of titrated oxygen treatment in patients with breathlessness and a history or clinical likelihood of chronic obstructive pulmonary disease in the prehospital setting.

Trial registration Australian New Zealand Clinical Trials Register ACTRN12609000236291.

\section{INTRODUCTION}

Chronic obstructive pulmonary disease is a major public health problem in many countries. The World Health Organization estimates that 210 million people have moderate to severe chronic obstructive pulmonary disease and that three million people died of the condition in 2005. ${ }^{1}$ Chronic obstructive pulmonary disease is associated with a substantial economic burden; estimates of cost include $\$ 49.9$ (£31.5; €36.3) billion in the United States in 2010 and $\$ 1.5$ billion in the United Kingdom in $2004 .^{23}$ The course of the disease is characterised by episodes, known as acute exacerbations, when symptoms of cough, sputum production, and breathlessness become much worse. These are a frequent cause of admission to hospital, estimated to cost approximately $\$ 73$ billion a year in the United States and $\$ 700$ million a year in Australia and Canada. ${ }^{4-6}$ Standard prehospital management of an acute exacerbation of chronic obstructive pulmonary disease includes nebulised bronchodilators (usually driven by $6-8 \mathrm{l} / \mathrm{min}$ of oxygen), corticosteroids, and oxygen.

Oxygen is commonly administered to patients with an acute exacerbation of chronic obstructive pulmonary disease, as well as for a range of other medical emergencies, ${ }^{78}$ and can save lives by preventing severe hypoxaemia. Administration of high flow oxygen to normal people leads to an increase in the minute ventilation (the volume of air inspired into or expired out 
of the lungs in one minute) and a decrease in end tidal carbon dioxide concentration. ${ }^{910}$ However, even in patients with stable chronic obstructive pulmonary disease, hyperoxia leads to a decrease in minute ventilation and an increase in transcutaneous carbon dioxide. ${ }^{11}$ These changes are postulated to occur as a result of either depression of ventilation or worsening inequality in ventilation-perfusion due to recruitment of poorly ventilated lung units (alveolus and associated pulmonary capillaries where gas exchange takes place) by reversal of local hypoxic pulmonary vasoconstriction subsequently releasing sequestered carbon dioxide. ${ }^{8}$ Oxygen induced changes in carbon dioxide concentrations in chronic obstructive pulmonary disease were first shown in $1955,{ }^{12}$ several years after a report on the deleterious effects of oxygen in this condition. ${ }^{13}$ Campbell was then fundamental in establishing the in-hospital practice of titrating oxygen treatment by using venturi masks. ${ }^{14}$ Subsequently, mortality in acute exacerbations of chronic obstructive pulmonary disease was reported to be associated with an increase in chronic stable levels of arterial carbon dioxide pressure, rather than the absolute level of hypercapnia. ${ }^{15}$

More recently, audits of hospital management have shown that the administration of high flow oxygen during acute exacerbations of chronic obstructive pulmonary disease has been associated with increases in mortality, length of hospital stay, requirement for ventilation, and admission to high dependency units. ${ }^{16-20}$ Furthermore, the use of titrated oxygen treatment has been associated with less acidosis, a lower requirement for assisted ventilation, and reduced mortality. ${ }^{1520} \mathrm{~A}$ one year prevalence study showed that the injudicious use of oxygen treatment caused acidosis in patients with acute exacerbations of chronic obstructive pulmonary disease, but a proportion of these patients were rapidly able to correct their $\mathrm{pH}$ once the fraction of inspired oxygen was reduced..$^{19}$ Despite the accumulating evidence, high flow oxygen treatment continued to be the standard of care in Australia and other countries up to the time this trial started. ${ }^{7172120}$ In recent years, awareness of the potential harm of routine use of high flow oxygen has increased, and attempts have been made to modify practice. Concern about the routine use of high flow oxygen to relieve breathlessness in emergency settings has prompted the release of the first international guidelines for the emergency use of oxygen, ${ }^{8}$ but the need for randomised controlled trials is widely acknowledged. ${ }^{816192223}$ A recent Cochrane review on oxygen treatment and acute exacerbations of chronic obstructive pulmonary disease in the prehospital setting identified two ongoing trials, reinforcing the medical community's recognition of the need for evidence in this area. ${ }^{22425}$

Despite published guidelines, the lack of clear evidence showing the benefit of titrated oxygen treatment may be responsible for the lack of widespread cultural change among practitioners, authorities, and opinion leaders. The difficulty in modifying practice in the prehospital setting is compounded by the lack of equipment capable of delivering controlled oxygen treatment while administering nebulised drugs in ambulances. This study was motivated by the need for quantitative evidence on the dangers of high flow oxygen, to support recommendations for the use of titrated oxygen treatment for patients with an acute exacerbation of chronic obstructive pulmonary disease. Our ultimate objective is to improve the quality of care for patients. The aim of this study was to compare standard high flow oxygen treatment with titrated oxygen treatment for patients with an acute exacerbation of chronic obstructive pulmonary disease in the prehospital setting.

\section{METHODS}

\section{Participants}

The study population comprised people aged 35 years or older with breathlessness and a history or risk of chronic obstructive pulmonary disease. Paramedics at the site of the emergency determined the diagnosis on the basis of appropriate acute symptoms, a history of chronic obstructive pulmonary disease (or emphysema) from the patient, or a greater than 10 pack year history of smoking. The study period was June 2006 to July 2007, and all participants were transported by ambulance and admitted to the Royal Hobart Hospital. This is located in the Tasmanian state capital of Hobart and is the major general hospital serving southern Tasmania (population approximately 250000 ).

\section{Randomisation}

The study was a randomised, controlled, parallel group trial with two arms: paramedics in the active arm administered titrated oxygen treatment and those in the control arm administered conventional high flow oxygen treatment. We invited paramedics from the Tasmanian Ambulance Service to participate in the study and used cluster randomisation to assign them to one of the treatment groups. We used computerised random number generation after stratification by rurality, to reduce differences associated with transportation time between urban and rural areas. This procedure ensured that treatment allocation was concealed before randomisation. Neither paramedics nor the research team were blinded to treatment after randomisation. In situations in which multiple paramedics attended a patient and had been allocated to different study arms, the paramedic responsible for treatment was to deliver oxygen according to his or her treatment allocation. We informed nursing and medical staff at the emergency department of Royal Hobart Hospital about the trial and asked them to take an arterial blood gas sample from each patient immediately on arrival. We asked paramedics to inform hospital staff on arrival that a patient was enrolled in the study and to request the same ongoing oxygen treatment until an arterial blood gas sample had been taken. Hospital staff were thus not blinded to the treatment allocation. The ethics committee waived patients' consent, for pragmatic reasons in an acute emergency situation and because high flow oxygen treatment was the default standard 
Table 1|Baseline characteristics of treating paramedics. Values are numbers (percentages) unless stated otherwise

\begin{tabular}{lcc}
$\begin{array}{l}\text { Characteristic } \\
\text { Location: }\end{array}$ & $\begin{array}{c}\text { Control (high flow oxygen) } \\
(\mathrm{n}=30)\end{array}$ & $\begin{array}{c}\text { Active (titrated oxygen) } \\
(\mathrm{n}=32)\end{array}$ \\
\hline Urban & $23(77)$ & $23(72)$ \\
\hline Rural & $7(23)$ & $9(28)$ \\
\hline Qualification: & $10(33)$ & $8(25)$ \\
\hline Paramedic & $20(67)$ & $24(75)$ \\
\hline Intensive care paramedic & $15.5(8.3)$ & $16.7(12.1)$ \\
\hline Mean (SD) years of experience & &
\end{tabular}

practice in the Tasmanian Ambulance Service when the study started. To avoid contamination across treatment groups, we chose paramedics rather than patients as the unit of randomisation.

\section{Interventions}

Patients in the active arm received titrated oxygen treatment delivered by nasal prongs to achieve arterial oxygen saturations between $88 \%$ and $92 \%$, with concurrent bronchodilator treatment administered by a nebuliser driven by compressed air (Walkie nebulisation air compressors, FlaemNova, Milan, Italy) and delivered via a facemask over the nasal prongs. The control arm received high flow oxygen treatment (8-10 l/min) administered by a non-rebreather face mask and bronchodilators delivered by nebulisation with oxygen at flows of $6-8 \mathrm{l} / \mathrm{min}$. Pulse oximeters were used to measure oxygen saturations and titrate oxygen to target saturations in the active arm. Oximeters were also used to measure oxygen saturation in the high flow oxygen arm. The oximeters were built into the MRL (Welch Allyn, New York, USA) and MRX (Phillips Medical Systems, Andover, USA), had no alarm boundaries programmed, and had a visual readout only. Oxygen saturations were recorded on the ambulance report form.

All patients received other standard treatment according to Tasmanian Ambulance Service guidelines, including basic support, nebulised bronchodilators (salbutamol $5 \mathrm{mg}$ made up in $2.5 \mathrm{ml}$ normal saline, ipratropium bromide $500 \mu \mathrm{g}$ made up with $2.5 \mathrm{ml}$ normal saline), dexamethasone $8 \mathrm{mg}$ intravenously, and, where necessary, salbutamol 200-300 mg intravenously or $500 \mathrm{mg}$ intramuscularly.

\section{Data collection}

Patients entered into the study were identified by two means. Paramedics annotated ambulance report forms to indicate patients who were entered into the trial to facilitate subsequent identification of these patients by the research team. The emergency department at Royal Hobart Hospital also supplied a list of all patients transported by ambulance with a respiratory diagnosis, which the research team cross checked against ambulance records to compile a complete list of patients included in the study. We retrospectively defined a subgroup with chronic obstructive pulmonary disease as those patients with a definite diagnosis of chronic obstructive pulmonary disease, as defined by national guidelines. ${ }^{5} \mathrm{~A}$ respiratory physician blind to treatment allocation reviewed lung function data and smoking history, from private and public medical records. Only lung function data recorded in the five years before study entry were considered. Patients with no lung function data or who did not fulfil spirometric criteria for chronic obstructive pulmonary disease were excluded from the subgroup analyses. Royal Hobart Hospital provided data on arterial blood gas results taken within 30 minutes of arrival at the emergency department, requirement for invasive (endotracheal intubation) or non-invasive ventilation, the length of stay in hospital, and mortality. Length of treatment in the ambulance and oxygen saturation during prehospital management came from the Tasmanian Ambulance Service's records. Data were missing from both the ambulance and hospital patient records, so the denominators vary among analyses.

\section{Statistical analysis}

We used the oxygen dissociation curve and Kelman's equation to determine whether blood gas samples were arterial or venous if this had not been recorded. ${ }^{26} \mathrm{We}$ compared the measured arterial oxygen concentrations with the concentrations calculated from pulse oximetry at the time of the blood gas sample. We took a difference greater than $5 \%$ between the calculated and measured oxygen concentrations to indicate that the blood gas was a venous sample. For venous samples, we used the formulations described by $\mathrm{Ak}$ to estimate arterial values for $\mathrm{pH}$, carbon dioxide, and bicarbonate. ${ }^{27} \mathrm{We}$ used non-paired Student's $t$ tests to compare arterial and converted venous blood gases. As no significant differences existed between arterial or converted venous samples for $\mathrm{pH}$, carbon dioxide, or bicarbonate, we combined them for subsequent analyses. We could not convert venous oxygen concentrations, as comparative arterial oxygen concentrations are not provided in the conversion equations, and we thus report only measured arterial oxygen concentrations.

The primary outcome was prehospital and in-hospital mortality. We used log binomial regression to compare the risk of death for patients in the two treatment arms. We compared secondary outcomes - namely, length of hospital stay and blood gas measurements $(\mathrm{pH}$, arterial carbon dioxide, bicarbonate, and arterial oxygen) - by using Student's $t$ tests after transformation to remove skewness. We combined ventilation categories (invasive and non-invasive), as the number of observations was small, and used log binomial regression to compare them. We based initial analyses on the intention to treat principle and restricted a further set of per protocol analyses to patients who received treatment that complied with the study protocol. We did all analyses for all study patients and for the subgroup of patients with chronic obstructive pulmonary disease confirmed by lung function tests. We adjusted standard errors to account for any correlation between observations for the same 
Table 2 |Baseline characteristics for all patients and subgroup with confirmed diagnosis of chronic obstructive pulmonary disease (COPD). Values are mean (SD) unless stated otherwise

\begin{tabular}{lcc}
$\begin{array}{l}\text { Characteristic } \\
\text { All patients }(n=405)\end{array}$ & Active (titrated oxygen) \\
\hline No (\%) male & $114 / 226(50)$ & $83 / 179(46)$ \\
\hline Age (years) & $69(10.9)(n=202)$ & $69(11.8)(n=152)$ \\
\hline Prehospital treatment time (minutes) & $47(19)(n=156)$ & $47(18)(n=144)$ \\
\hline Pretreatment oxygen saturation (\%) & $86(13.6)(n=189)$ & $88(9.8)(n=160)$ \\
\hline Confirmed diagnosis of COPD (n=214) & & $45 / 97(46)$ \\
\hline No (\%) male & $57 / 117(49)$ & $67.9(10.3)(n=97)$ \\
\hline Age (years) & $68.0(10.2)(n=117)$ & $43.3(16.5)(n=97)$ \\
\hline Per cent predicted FEV 1 & $42.1(16.4)(n=117)$ & $46.3(22.1)(n=83)$ \\
\hline Smoking history (pack years) & $45.5(26.0)(n=87)$ & $50(19)(n=80)$ \\
\hline Prehospital treatment time (minutes) & $47(17)(n=87)$ & $87(10)(n=87)$ \\
\hline Pretreatment oxygen saturation $(\%)$ & $84(14)(n=101)$ & \\
\hline
\end{tabular}

$\mathrm{FEV}_{1}=$ forced expiratory volume in one second.

paramedic (clustering). We used Intercooled Stata 10 for Windows for all analyses and considered a $\mathrm{P}$ value of 0.05 to be statistically significant.

For the primary outcome measure, mortality, we did power calculations assuming a $12 \%$ absolute difference in mortality between treatment arms, based on the study by Denniston et al. ${ }^{16} \mathrm{We}$ calculated that a total of 200 participants (100 in each of two equal sized groups) would provide $83 \%$ power to detect a $70 \%$ reduction in mortality, from $14 \%$ in the high flow oxygen treatment arm to $2 \%$ in the titrated oxygen treatment arm. This sample size would also provide $94 \%$ power to detect a difference in arterial carbon dioxide pressure of $10 \mathrm{~mm} \mathrm{Hg}$ (common SD $20 \mathrm{~mm} \mathrm{Hg}$ ) between the two groups. We allowed for a 25\% dropout rate and thus needed a minimum of 135 participants in each arm.

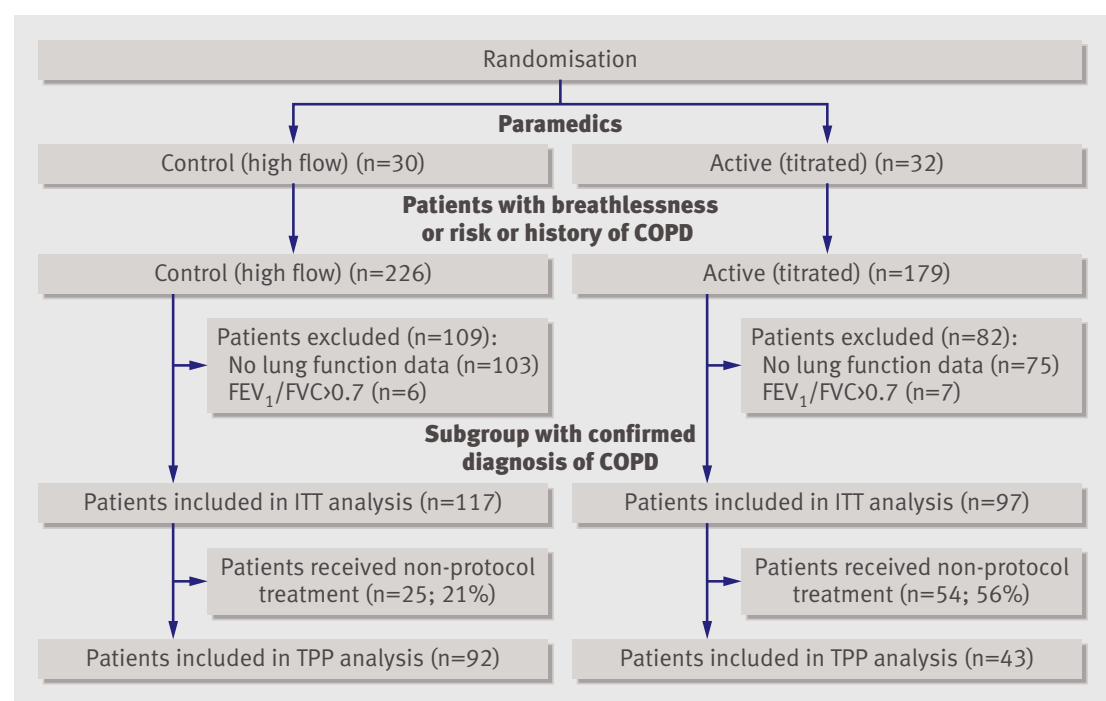

Flow of participants through study. $\mathrm{COPD}=$ chronic obstructive pulmonary disease; $\mathrm{FEV}_{1}=$ forced expiratory volume in one second; $F V C=$ forced vital capacity; ITT=intention to treat; TPP=treatment per protocol

\section{RESULTS}

We received consent to participate from $62(98 \%)$ of the 63 full time paramedic staff, who had a one month familiarisation and training period before data collection started. Paramedics were equally distributed between the two treatment arms with regard to rurality, training, and experience (table 1). The paramedics transported 405 eligible patients to the Royal Hobart Hospital during the 13 month study period: 226 were treated by paramedics assigned to the high flow oxygen arm and 179 by those in the titrated oxygen arm. The subgroup of patients with chronic obstructive pulmonary disease confirmed by lung function tests contained 214 of the study patients: 117 were treated by paramedics assigned to the high flow oxygen arm and 97 by those in the titrated oxygen arm (figure). Seventy-nine (37\%) randomised patients, 25 in the high flow arm and 54 in the titrated arm, did not receive treatment according to protocol, leaving 43 patients in the titrated oxygen arm and 92 patients in the high flow oxygen arm for the per protocol analyses.

Baseline characteristics for all patients and for the subgroup of patients with confirmed chronic obstructive pulmonary disease did not differ significantly between the treatment arms in terms of age, sex, initial oxygen saturation, or prehospital treatment time (table 2). Patients in the chronic obstructive pulmonary disease subgroup did not differ significantly between groups in per cent predicted forced expiratory volume in one second or smoking history (table 2). Blood gas samples were taken after arrival at hospital in only 122 $(57 \%)$ patients, and of these only $23(19 \%)$ were arterial samples drawn within 30 minutes of arrival.

In the intention to treat analysis for all patients, we found a significant difference between the two treatment arms for mortality (relative risk $0.42,95 \%$ confidence interval 0.20 to $0.89 ; \mathrm{P}=0.02$ ) (table 3 ). Mortality was $9 \%$ (21 deaths) in the high flow oxygen arm compared with $4 \%$ (seven deaths) in the titrated oxygen arm. In the intention to treat analysis for the subgroup with confirmed chronic obstructive pulmonary disease, we also found a significant difference in mortality: $9 \%$ (11 deaths) in the high flow arm compared with $2 \%$ (two deaths) in the titrated oxygen arm (relative risk $0.22,0.05$ to $0.91 ; \mathrm{P}=0.04$ ) (table 3). All deaths occurred after arrival at hospital, including two in the emergency department and two in the intensive care unit. Respiratory failure was the primary cause of death in all cases, and approximately $70 \%$ of deaths occurred within the first five days of admission for both treatment arms.

In the intention to treat analysis for secondary outcomes, we found no significant differences between treatment arms for length of hospital stay or requirement for ventilation for all patients or the subgroup with confirmed chronic obstructive pulmonary disease (table 3). Arterial blood gas measurements did not differ significantly between groups, although only about $11 \%$ of patients had these done within 30 minutes of arrival at hospital. The mean time to blood gas analysis for all samples (venous and arterial) was 1 hour 
Table $3 \mid$ Intention to treat analysis. Values are numbers (percentages) unless stated otherwise

\begin{tabular}{|c|c|c|c|c|}
\hline & Control (high flow oxygen) & Active (titrated oxygen) & Treatment effect & $P$ value \\
\hline \multicolumn{5}{|l|}{ Mortality } \\
\hline All patients & $21 / 226(9)$ & $7 / 179(4)$ & $0.42(0.20 \text { to } 0.89)^{\star}$ & 0.02 \\
\hline Confirmed COPD & $11 / 117(9)$ & $2 / 97(2)$ & $0.22(0.05 \text { to } 0.91)^{\star}$ & 0.04 \\
\hline \multicolumn{5}{|l|}{ Incidence of ventilation } \\
\hline All patients & $19 / 213(9)$ & $13 / 166(8)$ & $0.88(0.45 \text { to } 1.72)^{\star}$ & 0.70 \\
\hline Non-invasive ventilation & 7 & 8 & & \\
\hline Invasive ventilation & 12 & 5 & & \\
\hline Confirmed COPD & 15/105 (14) & $8 / 84(10)$ & $0.67(0.29 \text { to } 1.54)^{\star}$ & 0.34 \\
\hline Non-invasive ventilation & 6 & 5 & & \\
\hline Invasive ventilation & 9 & 3 & & \\
\hline \multicolumn{5}{|l|}{ Length of hospital stay (mean (SD) days) } \\
\hline All patients & $5.9(5.6)(n=226)$ & $5.5(5.9)(n=179)$ & $-0.45(0.57) \dagger$ & 0.19 \\
\hline Confirmed COPD & $6.3(5.8)(n=117)$ & $5.4(4.1)(n=97)$ & $-0.88(0.70) \dagger$ & 0.37 \\
\hline \multicolumn{5}{|c|}{ Arterial blood gases ( $\$ 30 \mathrm{~min}$ ) (confirmed COPD patients) } \\
\hline Mean (SD) pH & $7.29(0.14)(n=19)$ & $7.35(0.16)(n=19)$ & $0.06(0.05) \dagger$ & 0.11 \\
\hline Mean (SD) carbon dioxide (mm Hg) & $77.8(49.2)(n=20)$ & $54.7(31.1)(n=20)$ & $-23.1(13.0) \dagger$ & 0.06 \\
\hline Mean (SD) bicarbonate (mmol/l) & $32.3(10.1)(n=19)$ & $26.8(6.5)(n=19)$ & $-5.5(2.76) \dagger$ & 0.07 \\
\hline Mean (SD) oxygen (mm Hg) (arterial only) & $98.4(46.1)(n=14)$ & $79.3(24.9)(n=9)$ & $-19.1(16.8) \dagger$ & 0.34 \\
\hline
\end{tabular}

41 minutes after arrival at hospital for the high flow oxygen group and 1 hour 46 minutes for the titrated group. Blood samples for gas analysis were drawn from 59/97 $(61 \%)$ patients in the titrated oxygen arm and $63 / 117(54 \%)$ in the high flow arm.

In the per protocol analysis for the subgroup with confirmed chronic obstructive pulmonary disease, we found significant differences between treatment arms for blood gas measurements (table 4). Patients who received titrated oxygen were significantly less likely to have respiratory acidosis $(\mathrm{P}=0.01)$ due to acute hypercapnia $(\mathrm{P}=0.02)$ than were patients who received high flow oxygen. We found no significant differences between treatment arms in length of hospital stay or requirement for ventilation for all patients or the subgroup with confirmed chronic obstructive pulmonary disease (table 4). Death rates were similar to those seen in the intention to treat analysis for all patients and the subgroup with confirmed chronic obstructive pulmonary disease, although the differences did not reach statistical significance (table 4).

\section{DISCUSSION}

We found that oxygen treatment, titrated by paramedics to achieve arterial oxygen saturations between $88 \%$ and $92 \%$, for patients with breathlessness and a history or risk of chronic obstructive pulmonary disease, reduced the risk of death from respiratory failure by $58 \%$ for all patients and $78 \%$ for patients with confirmed chronic obstructive pulmonary disease, compared with high flow oxygen. For high flow oxygen treatment in patients with confirmed chronic obstructive pulmonary disease in the prehospital setting, the number needed to harm was 14; that is, for every 14 patients who are given high flow oxygen, one will die.
Patients with chronic obstructive pulmonary disease who received titrated oxygen according to the protocol were also significantly less likely to have respiratory acidosis or hypercapnia.

\section{Comparison with other studies}

This is the first randomised controlled trial of oxygen treatment for patients with a presumed acute exacerbation of chronic obstructive pulmonary disease in the prehospital setting. We used mortality as the primary outcome for the study because it is easily measured and is less subject to confounding than are other health outcomes such as length of stay in hospital. Our death rate of 9\% in the high flow oxygen arm is similar to that seen in audits of patients admitted to hospital with acute exacerbations of chronic obstructive pulmonary disease. ${ }^{2829}$ Importantly, our study provides much needed support for recent recommendations that titrated oxygen, administered to achieve arterial oxygen saturations between $88 \%$ and $92 \%$, should be used in emergency situations. ${ }^{819}$

In the treatment per protocol analyses, patients with chronic obstructive pulmonary disease who received high flow oxygen were more likely to have respiratory acidosis with a significantly higher arterial carbon dioxide pressure. This is consistent with previous studies that have found that increased carbon dioxide and respiratory acidosis are associated with poor outcomes, including increased mortality. ${ }^{151630}$ Reversal or prevention of these abnormalities by titrated oxygen has been associated with better in-hospital survival. ${ }^{193132}$ Although patients given high flow oxygen were more acidotic, the arterial oxygen pressure did not differ greatly between groups, which may reflect the more rapid change that occurs in response 


\begin{tabular}{|c|c|c|c|c|}
\hline & Control (high flow oxygen) & Active (titrated oxygen) & Treatment effect & $P$ value \\
\hline \multicolumn{5}{|l|}{ Mortality } \\
\hline All patients & 16/177 (9) & $3 / 66(5)$ & $0.50(0.16 \text { to } 1.54)^{\star}$ & 0.23 \\
\hline Confirmed COPD & 9/92 (10) & $1 / 43(2)$ & $0.24(0.04 \text { to } 1.57)^{\star}$ & 0.14 \\
\hline \multicolumn{5}{|l|}{ Incidence of ventilation } \\
\hline All patients & 19/167 (11) & $5 / 63(8)$ & $0.70(0.25 \text { to } 1.97)^{\star}$ & 0.50 \\
\hline Non-invasive ventilation & 7 & 4 & & \\
\hline Invasive ventilation & 12 & 1 & & \\
\hline Confirmed COPD & $15 / 83(18)$ & $3 / 40(8)$ & $0.42(0.14 \text { to } 1.20)^{*}$ & 0.11 \\
\hline Non-invasive ventilation & 6 & 2 & & \\
\hline Invasive ventilation & 9 & 1 & & \\
\hline \multicolumn{5}{|l|}{ Length of hospital stay (mean (SD) days) } \\
\hline All patients & $5.9(5.5)(n=177)$ & $6.0(5.3)(n=66)$ & $0.09(0.78) \dagger$ & 0.87 \\
\hline Confirmed COPD & $6.5(6.0)(n=92)$ & $6.2(4.6)(n=43)$ & $-0.29(1.04) \dagger$ & 0.96 \\
\hline \multicolumn{5}{|c|}{ Arterial blood gases ((30 min) (confirmed COPD patients) } \\
\hline Mean (SD) pH & $7.29(0.15)(n=18)$ & $7.41(0.09)(n=10)$ & $0.12(0.05) \dagger$ & 0.01 \\
\hline Mean (SD) carbon dioxide $(\mathrm{mm} \mathrm{Hg})$ & $76.5(50.2)(n=19)$ & $42.9(14.2)(n=10)$ & $-33.6(16.3) \dagger$ & 0.02 \\
\hline Mean (SD) bicarbonate $(\mathrm{mmol} / \mathrm{l})$ & $31.5(9.9)(n=18)$ & $26.0(4.2)(n=10)$ & $-5.5(3.30) \dagger$ & 0.15 \\
\hline Mean (SD) oxygen (mm Hg) (arterial only) & $98.4(46.1)(n=14)$ & $81.5(30.9)(n=6)$ & $-16.9(20.7) \dagger$ & 0.46 \\
\hline
\end{tabular}

to altering the fraction of inspired oxygen compared with $\mathrm{pH}$ and carbon dioxide.

We found that patients with an acute exacerbation of chronic obstructive pulmonary disease treated with titrated oxygen in the prehospital setting for an average time of 45 minutes were less likely to develop respiratory acidosis and die than were patients treated with high flow oxygen. Therefore, a dispersed population and lengthy treatment and transport times may maximise the potential adverse effects of high concentration oxygen treatment compared with areas and services that may have short treatment times. The population around Hobart is reasonably urbanised, but outlying rural areas give a rather mixed population, ideal for a generalisable study.

\section{Strengths and limitations of study}

One limitation of our trial was the lower than expected rate of adherence to study protocols, in both prehospital oxygen treatment and the measurement of arterial blood gases on arrival at hospital, showing the difficulties associated with modifying practice. Of the 214 patients with confirmed chronic obstructive pulmonary disease, ambulance records showed that $37 \%$ received treatment that did not comply with the study protocol $(56 \%$ in the titrated oxygen arm and $21 \%$ in the high flow oxygen arm). In the titrated oxygen arm, all protocol violations involved administration of high flow oxygen at some point during prehospital treatment. We expect that this would have minimised any treatment effect in the intention to treat analysis, but we still found a significant reduction in mortality for titrated oxygen treatment. The frequent lack of compliance in the titrated oxygen arm is probably a result of the entrenched culture and training in emergency medicine, which emphasises that high flow oxygen will save lives in acute respiratory emergencies by preventing severe hypoxaemia. From reviews of charts and interviews with paramedics, we found no evidence that the breaches of protocol were a result of malfunction of monitoring of saturation (oximeter), patients requesting more oxygen, or lack of understanding of the protocol. However, feedback indicated that some paramedics were concerned about insufficient delivery of oxygen in distressed patients, which suggests that they believed the entrenched cultural training that "more is better." This culture, combined with the absence of high quality evidence on the potential dangers of oxygen, may have been responsible for the ongoing practice of routine delivery of high flow oxygen. ${ }^{78161719-2133-35}$

A second limitation of this study was the low rate of arterial blood gas sampling for study patients. Although we informed staff in the emergency department staff of the importance of arterial samples for this trial, compliance was low and only $11 \%$ of arterial samples were drawn within 30 minutes of arrival. An audit of oxygen treatment in the prehospital setting by Wijesinghe et al also found a low rate of arterial blood gas sampling for patients with acute exacerbations of chronic obstructive pulmonary disease. ${ }^{20}$ The authors suggested several potential reasons for this, including refusal by patients, reluctance of the doctor to do the test, a perception by the doctor that the test is not indicated, or limited time and staff resources. ${ }^{20}$ In the emergency department at the time of the trial, the reasons given mainly related to limitations in staff time and resources but also to reluctance to do tests regarded as not clinically indicated. This supports again the difficulty in "real world" clinical trials of getting clinicians 


\section{WHAT IS ALREADY KNOWN ON THIS TOPIC}

Audits have shown increased mortality, acidosis, and hypercarbia in patients with acute exacerbations of chronic obstructive pulmonary disease treated with high flow oxygen

High flow oxygen is still used routinely in prehospital and hospital areas for breathless patients with chronic obstructive pulmonary disease

A "more is better" oxygen culture is strong in prehospital management

\section{WHAT THIS STUDY ADDS}

Titrated oxygen treatment reduces mortality, acidosis, and hypercarbia in patients with acute exacerbation of chronic obstructive pulmonary disease treated before arrival at hospital

The risk of death was reduced by $78 \%$ by use of titrated oxygen rather than high flow oxygen, with a number needed to harm of 14

These findings provide strong evidence that titrated oxygen treatment should be used for hypoxic or breathless patients with chronic obstructive pulmonary disease in prehospital settings acute exacerbation of chronic obstructive pulmonary disease. Our findings provide the first high quality evidence from a randomised controlled trial for the development of universal guidelines and support the British Thoracic Society's recent guidelines on acute oxygen treatment, which recommend that oxygen should be administered only at concentrations sufficient to maintain adequate oxygen saturations. Although our findings may need to be confirmed in larger studies across other health systems, implementation of the new guidelines will now be easier. However, resources for an aggressive campaign of education will still be needed to change the "more is better" oxygen culture that may ignore the potential dangers of hyperoxia.

We thank the patients who participated in the study, the clinical and clerical staff of the Tasmanian Ambulance Service and emergency department at the Royal Hobart Hospital, laboratory staff at the Royal Hobart Hospital, and members of the respiratory department at the Royal Hobart Hospital, whose participation made this project possible. Contributors: MAA contributed to the concept and design of the trial, recruitment and training of paramedics, implementation and management of the trial, analysis and interpretation of data, and writing of the report. KEW contributed to management of the trial, data management, data analysis and interpretation, and writing the report. LB advised on data analysis and interpretation and reviewed the report. EHW contributed to data interpretation and reviewed the report. RW-B contributed to the concept and design of the trial, data interpretation, and writing the report. MAA is the guarantor.

Funding: The Australian College of Ambulance Professionals (ACAP) provided funding. FlaemNova, Milan, Italy, donated Walkie nebulisation air compressors. Neither of the study sponsors had a role in study design; data collection, analysis, or interpretation; or the writing of the report. Competing interests: All authors have completed the Unified Competing Interest form at www.icmje.org/coi_disclosure.pdf (available on request from the corresponding author) and declare: no support from any organisation for the submitted work; no financial relationships with any organisations that might have an interest in the submitted work in the previous 3 years; no other relationships or activities that could appear to have influenced the submitted work.

Ethical approval: The Human Research Ethics Committee (Tasmania) Network approved this study (study number H0008413). Data sharing: The statistical code and dataset are available from the corresponding author at maaustin@utas.edu.au. tal oxygen management on measured outcomes. Review of the hospital records did not provide any further insight into the treatment after arrival in hospital. Titrated oxygen is the recommended treatment for breathless patients with a history or risk of chronic obstructive pulmonary disease at the Royal Hobart Hospital, but in practice, especially at the time of the trial, many of these patients received high flow oxygen on admission to hospital. However, any change in oxygen treatment that occurred after arrival in hospital would have reduced the differences between the treatment arms, thus underestimating the risk associated with high flow oxygen. Unfortunately, collection of data on in-hospital management was beyond the scope of the study, so we cannot dissect the effect of prehospital and in-hospital oxygen administration.

\section{Conclusions and policy implications}

This randomised controlled trial found that titrated oxygen treatment in the prehospital setting resulted in a 78\% reduction in the risk of in-hospital respiratory failure and subsequent mortality, compared with high flow oxygen treatment, and a decreased risk of hypercapnia and respiratory acidosis for patients with an

1 World Health Organization. Chronic obstructive pulmonary disease (COPD). 2010. www.who.int/respiratory/copd/en/.

2 American Lung Association. Chronic obstructive pulmonary disease COPD. 2010. www.lungusa.org/assets/documents/publications/ solddc-chapters/copd.pdf.

3 British Lung Foundation. Invisible lives: chronic obstructive pulmonary disease (COPD)—finding the missing millions. British Lung Foundation, 2007 (available at www.lunguk.org/Resources/ British\%20Lung\%20Foundation/Migrated\%20Resources/ Documents/I/Invisible\%20Lives\%20report.pdf).

4 Halpern MT, Stanford RH, Borker R. The burden of COPD in the USA results from the Confronting COPD Survey. Respir Med 2003;97:81-9S.

5 McKenzie D, Frith P, Burdon J, Town G. The COPDX plan: Australian and New Zealand guidelines for the management of chronic obstructive pulmonary disease. Med / Aust 2003;178:1-40S

6 Mittmann N, Kuramoto L, Seung SJ, Haddon JM, Bradley-Kennedy C, FitzGerald JM. The cost of moderate and severe COPD exacerbations to the Canadian healthcare system. Respir Med 2008;102:413-21.

7 Hale KE, Gavin C, O’Driscoll BR. Audit of oxygen use in emergency ambulances and in a hospital emergency department. Emerg Med 2008;25:773-6.

8 O'Driscoll BR, Howard LS, Davison AG. BTS guideline for emergency oxygen use in adult patients. Thorax 2008;63:vi1-68.

9 Becker HF, Polo O, McNamara SG, Berthon-Jones M, Sullivan CE. Effect of different levels of hyperoxia on breathing in healthy subjects. J Appl Physiol 1996;81:1683-90.

10 Lambertsen CJ, Kough RH, Cooper DY, Emmel GL, Loeschcke HH, Schmidt CF. Oxygen toxicity: effects in man of oxygen inhalation at 1 and 3.5 atmospheres upon blood gas transport, cerebral circulation and cerebral metabolism. J Appl Physiol 1953;5:471-86. 
11 Sassoon CSH, Hassell KT, Mahutte CK. Hyperoxic-induced hypercapnia in stable chronic obstructive pulmonary disease. Am Rev Respir Dis 1987;135:907-11.

12 Westlake EK, Simpson T, Kaye M. Carbon dioxide narcosis in emphysema. QJ Med 1955;24:155-73.

13 Donald K. Neurological effects of oxygen. Lancet 1949;257:1056-7.

14 Campbell E. The J Burns Amberson lecture: the management of acute respiratory failure. Am Rev Respir Dis 1967;96:626-39.

15 Jeffrey AA, Warren PM, Flenley DC. Acute hypercapnic respiratory failure in patients with chronic obstructive lung disease-risk-factors and use of guidelines for management. Thorax 1992;47:34-40.

16 Denniston A, O'Brien C, Stableforth D. The use of oxygen in acute exacerbations of chronic obstructive pulmonary disease: a prospective audit of pre-hospital and hospital emergency management. Clin Med 2002;2:449-51.

17 Joosten EA. The effects of oxygen therapy in patients presenting to an emergency department with exacerbation of chronic obstructive pulmonary disease. Med J Aust 2007;5:235-8.

18 Robinson T, Young I. The role of hypoventilation and ventilationperfusion redistribution in oxygen-induced hypercapnia during acute exacerbations of chronic obstructive pulmonary disease. Am J Respir Crit Care Med 2001;163:1524-9.

19 Plant PK, Owen JL, Elliott MW. One year period prevalence study of respiratory acidosis in acute exacerbations of COPD: implications for the provision of non-invasive ventilation and oxygen administration. Thorax 2000;55:550-4.

20 Wijesinghe M, Perrin K, Healy B, Hart K, Clay J, Weatherall M, et al. Pre-hospital oxygen therapy in acute exacerbations of chronic obstructive pulmonary disease. Intern Med / 2009;39:1-18.

21 Gooptu B, Ward L, Ansari SO, Eraut CD, Law D, Davison AG. Oxygen alert cards and controlled oxygen: preventing emergency admissions at risk of hypercapnic acidosis receiving high inspired oxygen concentrations in ambulances and A \& E departments. Emerg Med 2006;23:636-8.

22 Austin M, Wood-Baker R. Oxygen therapy in the pre-hospital setting for acute exacerbations of chronic obstructive pulmonary disease. Cochrane Database Syst Rev 2006;3:CD005534.

23 Hurd S, Pauwels R. Global initiative for chronic obstructive lung disease (GOLD). Pulm Pharmacol Ther 2002;14:353-5.

24 Elliott DMWE. A prospective randomised controlled trial of oxygen targeted to maintain an oxygen saturation between 88 and $92 \%$ compared with standard therapy for patients with COPD during ambulance transfer to hospital. Leeds Teaching Hospitals NHS Trust, 2004.

25 Eiser DN. Comparison of controlled oxygen with standard oxygen therapy for COPD patients during ambulance transfer to hospital. London Ambulance Service NHS Trust, 2004.

26 Lumb A, Pearl R. Nunn's applied respiratory physiology. 6th ed Elsevier, 2005.

27 Ak A, Ogun CO, Bayir A, Kayis SA, Koylu R. Prediction of arterial blood gas values from venous blood gas values in patients with acute exacerbation of chronic obstructive pulmonary disease. Tohoku J Exp Med 2006;210:285-90.

28 Gunen H, Hacievliyagil SS, Kosar F, Mutlu LC, Gulbas G, Pehlivan E, et al. Factors affecting survival of hospitalised patients with COPD. Eur Respir J 2005;26:234-41.

29 Price LC, Lowe D, Hosker HS, Anstey K, Pearson MG, Roberts CM. UK national COPD audit 2003: impact of hospital resources and organisation of care on patient outcome following admission for acute COPD exacerbation. Thorax 2006;61:837-42.

30 Soler-Cataluna Jl, Martinez-Garcia MA, Sanchez PR, Salcedo E, Navarro M, Ochando R. Severe acute exacerbations and mortality in patients with chronic obstructive pulmonary disease. Thorax 2005;60:925-31.

31 Plant PK, Owen JL, Elliott MW. Non-invasive ventilation in acute exacerbations of chronic obstructive pulmonary disease: long term survival and predictors of in-hospital outcome. Thorax 2001;56:708-12.

32 Jeffrey AA, Warren PM, Flenley DC. Acute hypercapnic respiratory failure in patients with chronic obstructive lung disease: risk factors and use of guidelines for management. Thorax 1992;47:34-40.

33 New A. Oxygen: kill or cure? Pre-hospital hyperoxia in the COPD patient. Emerg Med J 2005;23:144-6.

34 Scales DC, Adhikari NKJ. Lost in (knowledge) translation: "all breakthrough, no follow through"? Crit Care Med 2008;36:1654-5.

35 Grol R, Grimshaw I. From best evidence to best practice: effective implementation of change in patients' care. Lancet 2003;362:1225-.30.

Accepted: 19 August 2010 